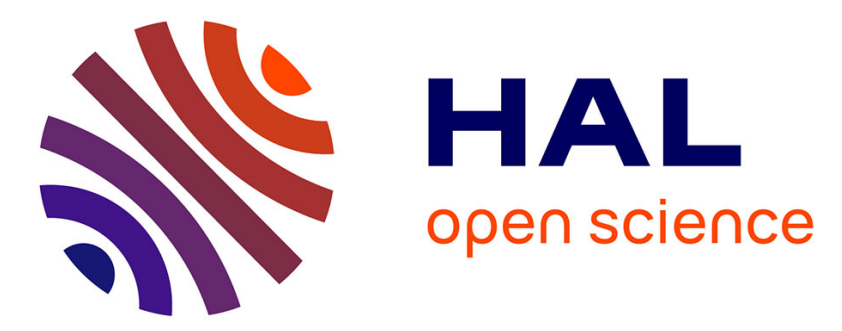

\title{
Intérêt d'un modèle hydrodynamique en deux dimensions pour interpréter le comportement des poissons dans les grands cours d'eau
}

H. Capra, E. Mcneil, M.C. Bouillon, H. Pella, C. Alfaro

\section{To cite this version:}

H. Capra, E. Mcneil, M.C. Bouillon, H. Pella, C. Alfaro. Intérêt d'un modèle hydrodynamique en deux dimensions pour interpréter le comportement des poissons dans les grands cours d'eau. La Houille Blanche - Revue internationale de l'eau, 2011, 6, p. 28 - p. 33. 10.1051/lhb/2011058 . hal-00727368

\section{HAL Id: hal-00727368 \\ https://hal.science/hal-00727368}

Submitted on 3 Sep 2012

HAL is a multi-disciplinary open access archive for the deposit and dissemination of scientific research documents, whether they are published or not. The documents may come from teaching and research institutions in France or abroad, or from public or private research centers.
L'archive ouverte pluridisciplinaire HAL, est destinée au dépôt et à la diffusion de documents scientifiques de niveau recherche, publiés ou non, émanant des établissements d'enseignement et de recherche français ou étrangers, des laboratoires publics ou privés. 


\title{
Intérêt d'un modèle hydrodynamique en deux dimensions pour interpréter le comportement des poissons dans les grands cours d'eau
}

\author{
Hervé CAPRA ${ }^{1}$, Eric McNEIL², Marie-Claire BOUILLON², Hervé PELLA¹, Carola ALFARO ${ }^{3}$ \\ 1. Cemagref, UR MAEP, Equipe Dynam - e-mail : herve.capra@cemagref.fr \\ 2.HydroQuébec - Production - e-mail : mcneil.eric@hydro.qc.ca \\ 3.AECOM - e-mail : carola.alfaro@aecom.com
}

\begin{abstract}
RESUME. - Cet article vise à décrire en quoi un outil performant de caractérisation des conditions abiotiques (modèle hydrodynamique en deux dimensions en l'occurrence) permet d'aborder des questions écologiques originales, comme une étude in situ des réponses comportementales des poissons du Rhône soumis à d'importantes variations des conditions environnementales. Dans un milieu aussi complexe (nombreux paramètres environnementaux qui interagissent) que le Rhône, les résultats présentés ont montré qu'une modélisation en deux dimensions permettait de prendre en compte, pour chaque période d'observation du milieu, la fluctuation des conditions d'écoulement, d'habitat et de température de l'eau. La mise en œuvre d'une telle approche constitue un atout majeur pour la compréhension de la structuration des communautés aquatiques en générale, et de poissons en particulier, trop rarement appliquée en France. Pour la première fois, les conditions environnementales vécues par les poissons au cours de leur déplacement ont été caractérisées. Cette approche peut également être appliquée pour simuler les conditions d'habitat et de température au cours de périodes significativement plus longues, compatibles, par exemple, avec le suivi de la structure de la communauté piscicole du Rhône entamé en 1979. En cela la mise en œuvre d'une modélisation hydrodynamique en 2D répond à l'une des questions majeures formulées par les experts dans la synthèse du projet Rhône Thermie - phase III en 2006. La disponibilité d'une description en deux dimensions, à des échelles spatiales et temporelles adéquates, et ce, pour une large gamme de débits susceptibles d'être observés dans le secteur d'étude, des conditions d'habitat et de température laisse supposer qu'il sera possible de connaître l'influence de ces deux facteurs environnementaux déterminants sur les choix comportementaux des poissons.
\end{abstract}

Mots clefs : modèle hydrodynamique en 2D, habitat, poissons, Rhône, variabilité, comportement

\section{Relevance of 2D hydraulic model to address fish behaviour in large rivers}

\begin{abstract}
The article presented herein seeks to demonstrate how a two-dimensional hydrodynamic model representing abiotic conditions can be used to study the behaviour of fish affected by large fluctuations of environmental conditions in the Rhône River. Despite the complexity of the Rhône's physical environment, the results presented in this article show that a 2D model is capable of correctly representing the hydraulic conditions, water temperature and habitats for the studied area at each time step. This represents a major advancement in the understanding of aquatic community structures and in particular, for the structure of fish communities that are rarely used in France. For the first time, the environmental conditions affecting fish and their spatial localization will be known simultaneously. The model can also simulate habitat conditions and temperatures over longer periods of time. For example, this methodology is compatible with the monitoring of fish communities of the Rhône initiated in 1979. In this regard, a 2D hydrodynamic model answered a major need of experts working on the Rhône therm - Phase III project in 2006. The interest in modeling precise habitat and temperature conditions in two-dimensions for a large array of flows allows one to speculate that it will be possible to know the role that these two key environmental factors play on the behavioural choices of fish.
\end{abstract}

Key words : 2D hydrodynamic model, habitat, fish, Rhône River, variability, behaviour

\section{INTRODUCTION}

Les ressources hydriques du fleuve Rhône sont exploitées pour différents usages, notamment la production hydroélectrique, le refroidissement des Centres Nucléaires de Production Electrique (CNPE) ainsi que l'approvisionnement en eau. Ces différentes utilisations de l'eau impliquent des modifications plus ou moins significatives du régime hydrologique et donc des conditions abiotiques qui y sont associées.

La production hydroélectrique génère des fluctuations de débit significatives sur de courts laps de temps (infra-journaliers) qui ne s'observent pas dans un régime hydrologique naturel et qui pourraient être assimilées à des éclusées. Ces fluctuations de débit se déclinent, en terme d'habitat du poisson, par des variations importantes de la disponibilité en habitat favorable.

Par ailleurs, l'eau du Rhône est également utilisée pour le refroidissement des réacteurs des CNPE. Il en résulte des rejets d'eau chaude (supérieurs de plusieurs degrés à la température de l'eau pompée) dans le Rhône, ce qui constitue un autre facteur environnemental susceptible d'influencer les communautés de poissons.

L'autorisation de rejet d'eau chaude accordée par les services de l'état oblige les CNPE à organiser des suivis des 
communautés aquatiques pour évaluer les conséquences du réchauffement de l'eau sur le fonctionnement écologique de l'écosystème perturbé. Pour le CNPE de Bugey en particulier, après 20 années d'étude, les conclusions et les recommandations du groupe de travail 'Etude Rhône Thermie - Phase III' (EDF, Drire, Agence de l'Eau, Diren, Univ. Lyon I, Aralep, Cemagref Aix et Lyon) illustraient clairement le besoin de prendre en compte conjointement la variabilité spatio-temporelle des conditions de débit, d'habitat et de température pour étudier la variabilité de la structure des communautés vivantes du Rhône (poissons, invertébrés, plantes ; Carrel et al., 2006 [1]). La connaissance des réponses des poissons à des variabilités de débit sous différentes conditions de température constitue une question d'intérêt primordial. L'approche de simulation numérique des conditions abiotiques susmentionnées a été identifiée comme la plus appropriée pour représenter la variabilité spatio-temporelle des conditions d'habitat et de la température. De ces constats est né le projet Bugey2D, initié en 2006, dont les objectifs principaux étaient :

- de caractériser précisément les variations spatio-temporelles des conditions de vie des communautés aquatiques du Rhône (habitat et température) ;

- d'analyser les réponses comportementales des poissons soumis à des variations simultanées et contrastées d'habitat et de température ;

L'objectif global du projet est d'étudier comment la sélection de l'habitat par les poissons peut être influencée par la température de l'eau, notamment dans un contexte perturbé tant d'un point de vue hydrologique que thermique (e.g. le Rhône à la hauteur de la centrale nucléaire de Bugey). L'hétérogénéité spatiale de l'habitat et de la température peuvent jouer un rôle significatif pour certaines espèces de poissons, notamment celles en marge de leurs tolérances. Pour connaître les stratégies d'utilisation de l'habitat par les poissons, une expérimentation a permis de suivre le comportement de 61 poissons (par télémétrie acoustique) pendant 3 mois au cours de l'été 2009 (Bergé et al., soumis [2]) dans le Rhône au droit du CNPE de Bugey.

Le présent article vise à montrer en quoi un outil performant de représentation des conditions abiotiques (modèle hydrodynamique en deux dimensions en l'occurrence) permet d'aborder des questions écologiques originales, comme une étude in situ des réponses comportementales des poissons du Rhône soumis à de fortes variations des conditions environnementales. Les techniques de simulation de l'habitat des poissons en fonction du débit sont des outils fréquemment utilisés dans le cadre d'expertises de gestion (e.g. définition de régime de débit réservé ; Reiser et al., 1989[3] ; Tharme, 2003[4]) mais beaucoup plus rarement pour étudier les relations entre des caractéristiques du milieu (e.g. disponibilité d'habitat) et le comportement des poissons comme par exemple l'étude de bioénergétique présentée par Hayes et al. (2007) [5].

\section{MATERIEL ET METHODE}

\section{II.1. Présentation du site}

Le secteur du Rhône choisi pour l'étude est situé au milieu du plus long tronçon du Rhône français non dérivé par une installation hydroélectrique. Ce tronçon, long de 36 kilomètres, est localisé à l'Est de Lyon, entre les barrages de Villebois (Sault-Brénaz) à l'amont et de Jons à l'aval (figure 1). Le site étudié s'étend sur 3,5 km entre l'amont (pk47) et l'aval (pk43,5) du CNPE de Bugey (EDF). La largeur du lit mineur sur le secteur est d'environ $140 \mathrm{~m}$. Les débits du Rhône sont essentiellement régulés par les barrages situés en amont du tronçon (notamment Seujet et Verbois, en Suisse, et Génissiat) et sont compris $90 \%$ du temps entre 160 et $742 \mathrm{~m}^{3} \cdot \mathrm{s}^{-1}$ pour un module de l'ordre de $473 \mathrm{~m}^{3} \cdot \mathrm{s}^{-1}$ (www.rdbrmc.com; à Lagnieu; débit médian de $\left.470 \mathrm{~m}^{3} \cdot \mathrm{s}^{-1}\right)$. La température journalière moyenne de l'eau du Rhône en amont du site d'étude, évaluée sur la période 1980-2009, est de $12,1^{\circ} \mathrm{C}$. Au cours de cette période, la température moyenne journalière de l'eau a fluctué entre $1{ }^{\circ} \mathrm{C}$ et $25,8^{\circ} \mathrm{C}$.

\section{II.2. Mise en œuvre du modèle hydrodynamique}

Une schématisation bidimensionnelle a été retenue pour caractériser les conditions abiotiques qui prévalent au sein du site à l'étude. Ce choix résulte des considérations suivantes : 1) les modèles de préférence d'habitat des poissons sont définis en fonction de vitesses moyennes sur la colonne d'eau ; 2) il n'y a pas de stratification verticale de la température de l'eau ; 3) il convient de disposer d'une description des conditions abiotiques avec une configuration et une résolution spatiale compatible avec une étude comportementale des poissons (ce que n'aurait pas permis d'atteindre le recours à une schématisation unidimensionnelle; les poissons étant susceptibles de se déplacer tant latéralement que longitudinalement par rapport au cours d'eau), et 4) une approche de modélisation hydrodynamique en trois dimensions n'aurait pas, dans le contexte du projet, apporté davantage d'information, tout en s'avérant plus complexe à mettre en œuvre et à valider.

La discrétisation géométrique du tronçon à l'étude a été réalisée sous la forme d'un maillage irrégulier d'éléments triangulaires en tenant notamment compte de la configuration bathymétrique du secteur (recours, à titre d'exemple, à un maillage de Delaunay contraint au droit de certaines sections de contrôle de l'écoulement à faibles débits). Le tronçon considéré pour les simulations numériques des conditions abiotiques a été étendu vers l'amont par rapport aux

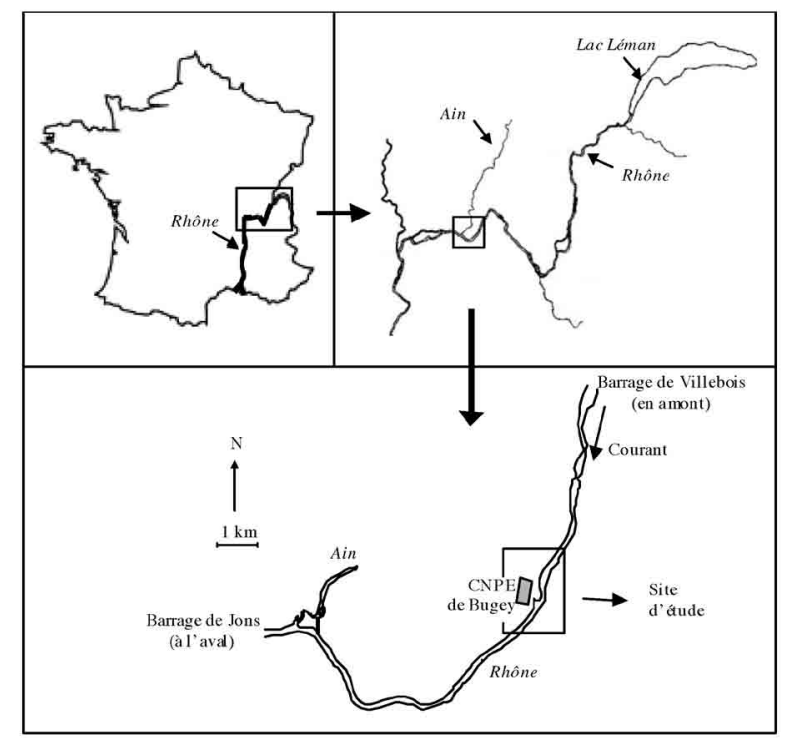

Figure 1 : Site d'étude, localisé sur le Rhône en amont de Lyon, à la hauteur du CNPE de Bugey. 
limites de la zone à l'intérieur de laquelle l'analyse comportementale du poisson est visée, ceci afin de palier aux risques d'introduction d'un biais lié à l'hypothèse de la distribution du débit spécifié à titre de condition à la frontière amont du domaine de calcul. L'attribution des élévations caractéristiques du lit du cours d'eau a bénéficié des efforts déployés à la caractérisation des élévations du lit mineur et des berges du secteur étudié.

Pour obtenir une forte densité de point de bathymétrie, les levés bathymétriques ont été réalisés avec un sondeur multi-faisceaux (Pella et al., 2008 [6]). Des données complémentaires de bathymétrie et de topographie ont été récoltées avec des techniques plus classiques (tachéomètre, DGPS et sondeur mono faisceau), et à partir de relevés LiDAR mis en œuvre par l'IGN pour l'Agence de l'Eau Rhône, Méditerranée et Corse en 2009. Les données ont été géo-référencées en utilisant la projection Lambert II étendu, généralement utilisée pour l'ensemble de la France Métropolitaine (projection conique conforme). Les coordonnées de chaque point sont considérées «centimétriques", ce qui signifie que, compte tenu des techniques utilisées pour les recueillir, les écarts entre les valeurs mesurées et la réalité sont de l'ordre de quelques centimètres.

La caractérisation des conditions de substrat prévalant au droit de chaque nœud du maillage a été établie à partir d'une cartographie de la répartition spatiale du substrat au sein du tronçon étudié. Cette cartographie a été élaborée en se basant sur des données acquises à partir d'observations directes depuis un bateau de même qu'à partir d'analyses de photos aériennes prises à très bas débit en hiver, garantissant ainsi une visibilité maximale.

Une attention particulière a été portée au processus d'étalonnage et de validation du modèle numérique d'écoulement, y compris au niveau de l'acquisition des données requises pour la réalisation de ces processus. A cet effet, une procédure permettant de varier les coefficients de friction le long du tronçon à l'étude en fonction du débit qui y transite a été implantée. Pour les processus d'étalonnage et de validation, des relevés de niveaux d'eau en fonction du débit ont été effectués au droit de six stations limnimétriques implantées sur les berges le long du tronçon étudié pour une large gamme de débits susceptibles d'y prévaloir. Pour chaque station limnimétrique, une relation niveau-débit a été établie pour une gamme de débit variant de 150 à $1100 \mathrm{~m}^{3} \cdot \mathrm{s}^{-1}$. Les relevés réalisés au droit de la station limnimétrique située à l'extrémité aval du tronçon à l'étude ont été exploités, compte tenu du caractère fluvial des écoulements prévalant dans le secteur, pour le traitement de la condition spécifiée à la frontière aval du tronçon; un hydrogramme étant spécifié à titre de condition à la frontière amont. Par ailleurs, des profils longitudinaux de surface libre (mesure du niveau d'eau en continu dans l'axe principal du fleuve) pour différents débits stabilisés ( 6 relevés entre 190 et $600 \mathrm{~m}^{3} \cdot \mathrm{s}^{-1}$ ) ont été relevés entre l'amont et l'aval du secteur d'étude.

La répartition transversale des vitesses moyennes d'écoulement sur la colonne d'eau a été estimée à plusieurs débits $\left(190\right.$ à $\left.600 \mathrm{~m}^{3} \cdot \mathrm{s}^{-1}\right)$ dans $\mathrm{l}^{\prime}$ 'axe de six sections transversales réparties le long du tronçon à l'étude. Les enregistrements étaient effectués à l'aide d'un ADCP (Rio Grande 1200 Khz- RD Instruments) couplé à un système DGPS (Leïca 1200) pour le géoréférencement. De la même manière, la température de l'eau du Rhône (à $0,5 \mathrm{~m}$ sous la surface) a été mesurée (sonde de type NKE SP2T10) dans l'axe de trois sections transversales pour différentes conditions de débit (450 à $\left.600 \mathrm{~m}^{3} \cdot \mathrm{s}^{-1}\right)$ et de température de l'eau à l'amont du site $\left(19,4\right.$ à $22,7^{\circ} \mathrm{C}$; Capra et al., 2008 [7]).
Une partie des données susmentionnées de conditions d'écoulement et de température de l'eau ont servi à l'étalonnage des modèles numériques d'écoulement et de température de l'eau. Les données n'ayant pas servi à l'étalonnage ont été utilisées pour la validation des processus d'étalonnage.

Au cours des relevés, les débits ont été mesurés avec un ADCP ou estimés à partir des débits déterminés par la Compagnie Nationale du Rhône au droit du site d'étude (pk 44,5). Certains relevés de niveaux d'eau ont été couplés à des mesures de débit au même moment.

Les résultats des simulations hydrodynamiques permettent de caractériser, pour chaque nœud du maillage, la vitesse moyenne d'écoulement et la profondeur prévalant localement en fonction du débit transitant par le tronçon à l'étude. Les conditions hydrodynamiques ainsi simulées servent d'intrants, avec les conditions de fonctionnement du CNPE (nombre de groupes en fonction), aux simulations de la répartition spatiale des températures de l'eau. Enfin, les conditions d'habitat ont été déterminées en considérant les courbes de préférences définies par Lamouroux et al. (1999) [8]. Les résultats de simulation d'habitat se présentent sous la forme d'un indice de qualité d'habitat défini en fonction de la profondeur et de la vitesse moyenne d'écoulement de même que du substrat dominant prévalant localement. Ces résultats se présentent notamment sous la forme d'une cartographie de la répartition spatiale des indices de qualité d'habitat associés à une phase du cycle vital et à un stade de développement d'une espèce piscicole. La disponibilité d'habitat associée au tronçon à l'étude est déterminée par la somme, pour l'ensemble des nœuds, de la superficie qui leur est associée (polygone de Thiessen) pondérée par l'indice de qualité d'habitat, lequel varie entre 0 et 1 . Cette surface pondérée utile (SPU) correspond donc à une représentation quantitative (exprimée en $\mathrm{m}^{2}$ ) de l'habitat piscicole disponible pour une phase donnée du cycle vital de l'espèce (stade de développement) considérée. Les conditions d'habitat et la disponibilité d'habitat ont été évaluées pour le barbeau, le gardon et la loche franche. Une description des cartographies d'habitat et de température de l'eau permettra une première évaluation des conditions environnementales qui prévalent au droit du CNPE.

Les simulations hydrodynamiques, thermiques et des conditions d'habitat ont été réalisées pour une gamme de débit variant de 150 à $750 \mathrm{~m}^{3} \cdot \mathrm{s}^{-1}$, laquelle correspond aux fluctuations typiques des débits durant la période d'enregistrement des déplacements des poissons au sein du site d'étude. Ces simulations permettent, pour les conditions de fluctuation de débit considérées, des visualisations en plan (cartographies) de la répartition spatiale des différentes variables simulées (la cartographie du substrat étant définie non variable en fonction du débit) et de connaître pour chaque localisation de poisson dans le site d'étude, les conditions d'habitat (e.g. hauteur d'eau, vitesse, substrat, température) utilisées par le poisson localisé.

\section{RESULTATS}

Un modèle de discrétisation géométrique du tronçon à l'étude a été constitué sous la forme d'un maillage irrégulier d'éléments triangulaires défini par 15157 nœuds et 29694 éléments. Les élévations aux nœuds du maillage ont été évalués à partir de 332864 points de mesure de l'élévation du lit mineur et des berges du secteur étudié. 
Le modèle hydrodynamique a été étalonné pour des débits de $190,300,400,525,600$ et $850 \mathrm{~m}^{3} \cdot \mathrm{s}^{-1}$, ce qui correspond à une large gamme de débits à l'intérieur de laquelle l'analyse du comportement du poisson est visée. Des écarts inférieurs à $1 \mathrm{~cm}$ entre les données simulées et mesurées ont été visés et obtenus au terme de l'étalonnage réalisé pour les différents débits susmentionnés.

Une vérification de la consistance entre les vitesses d'écoulement simulées et mesurées a également été effectuée pour les débits de 190 et $600 \mathrm{~m}^{3} \cdot \mathrm{s}^{-1}$. Cette vérification montre, d'une part, une concordance entre la configuration des champs de vitesses mesurés et simulés et, d'autre part, que les écarts des normes des vitesses n'excèdent pas $0,1 \mathrm{~m} \cdot \mathrm{s}^{-1}$ sur l'ensemble du site à l'étude.

Le processus d'étalonnage a fait l'objet d'une validation en considérant une gamme de débits variant de 150 à $725 \mathrm{~m}^{3} \cdot \mathrm{s}^{-1}$ (débits de $150,245,350,500$ et $725 \mathrm{~m}^{3} \cdot \mathrm{s}^{-1}$ ). Les écarts observés entre les niveaux simulés et mesurés dans le cadre de la validation se sont avérés de l'ordre du centimètre pour l'ensemble des profils longitudinaux à l'exception d'un point localisé $2 \mathrm{~km}$ en amont du secteur d'étude du comportement des poissons où l'écart s'est chiffré à $2 \mathrm{~cm}$ aux débits de 150 et de $245 \mathrm{~m}^{3} \cdot \mathrm{s}^{-1}$.

La figure 2 permet de comparer les conditions d'écoulement pour deux débits $\left(241\right.$ et $\left.725 \mathrm{~m}^{3} \cdot \mathrm{s}^{-1}\right)$. Leur examen souligne l'amplitude des fluctuations en terme de vitesse et de profondeur d'écoulement que sont susceptibles d'expérimenter les poissons sur des laps de temps très courts (de l'ordre de quelques heures). Le détail de l'évolution de la hauteur d'eau et de la bordure du lit mouillé lors d'une diminution de débit entre 725 et $241 \mathrm{~m}^{3} \cdot \mathrm{s}^{-1}$ est illustré sur la figure 3 . Ce résultat permet d'évaluer la quantité d'habitat exondé lors de telles diminutions de débit du Rhône à Bugey, et la discontinuité de l'accès aux abris situés en berge qui en résulte (cf. photo sur la figure 3 ).
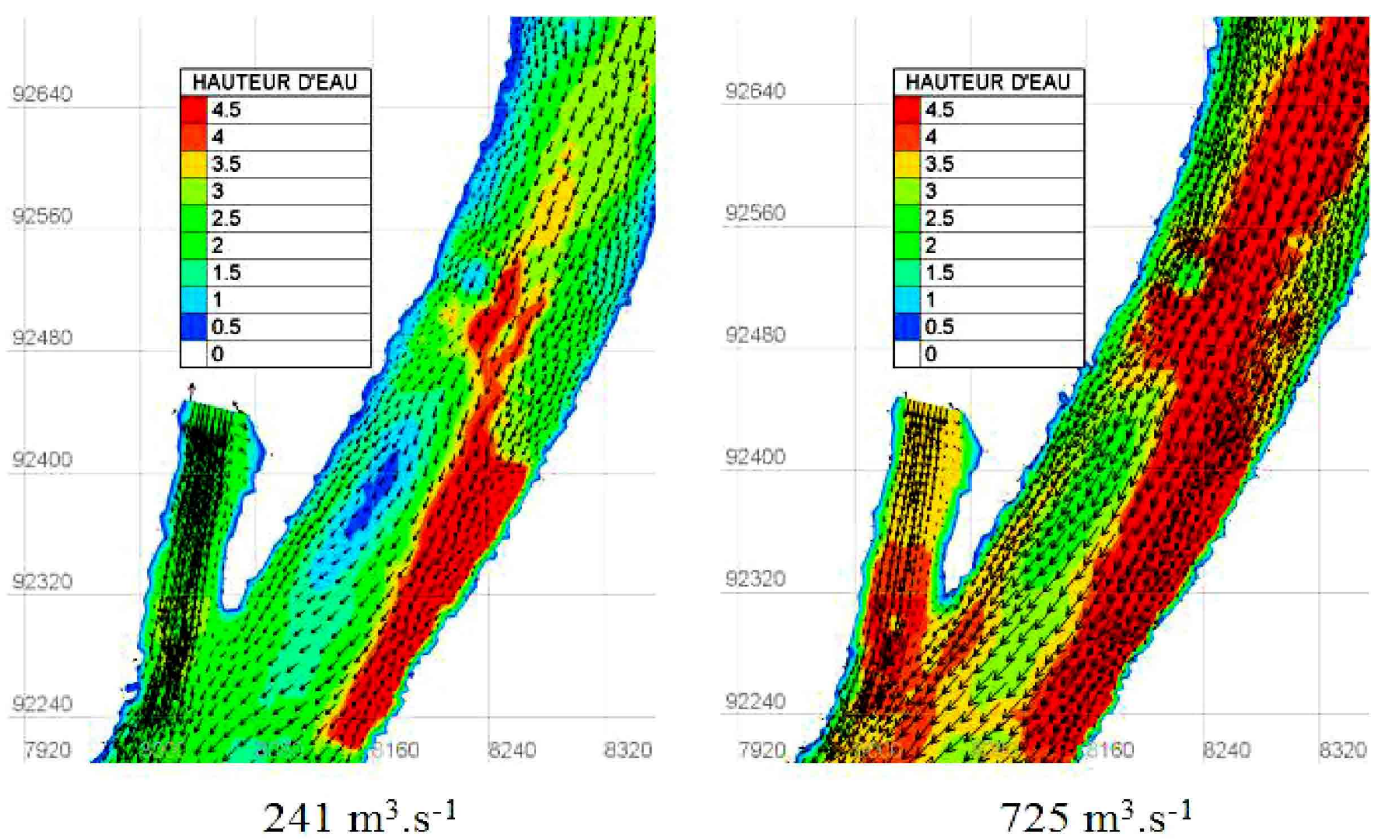

Figure 2 : Représentation des conditions d'écoulement (hauteur d'eau (m) en surface de couleur, et vitesse de courant, en vecteurs de taille proportionnelle au module de la vitesse) pour $241 \mathrm{~m}^{3} \cdot \mathrm{s}^{-1}$ et $725 \mathrm{~m}^{3} \cdot \mathrm{s}^{-1}$.

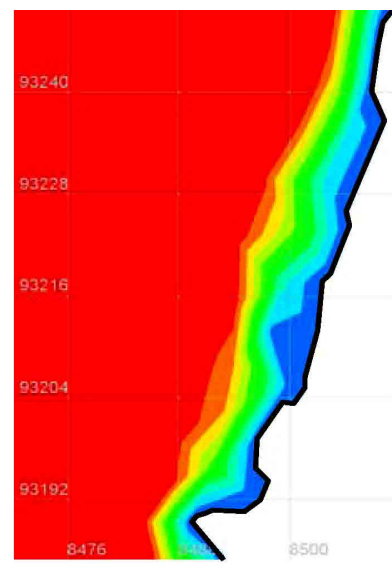

$725 \mathrm{~m}^{3} \cdot \mathrm{s}^{-1}$

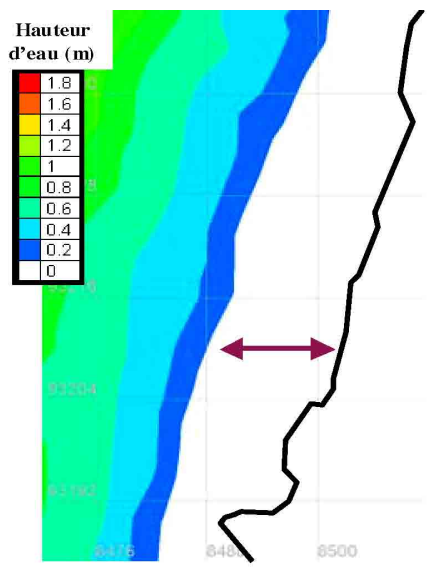

$241 \mathrm{~m}^{3} \cdot \mathrm{s}^{-1}$

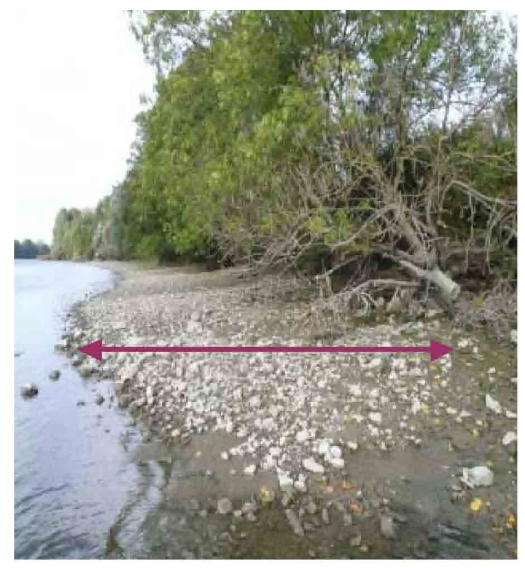

$170 \mathrm{~m}^{3} \cdot \mathrm{s}^{-1}$

Figure 3 : Évolution de la hauteur d'eau entre 725 et $241 \mathrm{~m}^{3} . \mathrm{s}^{-1}$ en bordure du lit mouillé illustrant la perte d'habitat disponible pour les poissons et la discontinuité de l'accès aux abris (cf. photo à droite). 
La simulation de la quantité d'habitat favorable potentiel (Surface Pondérée Utile en $\mathrm{m}^{2}$ ) en fonction du débit semble peu sensible au choix du débit d'étalonnage du modèle hydrodynamique (figure 4). Les courbes établies à partir des cinq débits d'étalonnage différents sont très proches, malgré un léger décalage à partir de $500 \mathrm{~m}^{3} \cdot \mathrm{s}^{-1}$.

Le modèle hydrodynamique mis en œuvre sur le Rhône à Bugey simule les conditions d'habitat utilisées par les poissons pour chacune de leurs localisations (figure 5). La création de chronogrammes permet de visualiser dans le temps et simultanément les profondeurs, les vitesses de cou- rant, les températures ou le substrat utilisés par les poissons et les conditions disponibles (e.g. le débit, dans la figure 5) dans le milieu.

Enfin, le modèle numérique de température de l'eau a également fait l'objet d'un étalonnage et d'une validation. Il en résulte des écarts maximaux de 1 'ordre de $0,5^{\circ} \mathrm{C}$ dans le cadre de la phase de validation. Par ailleurs, les résultats de simulation de température de l'eau ont été comparés à une cartographie des rejets d'eau chaude observés par le biais d'une thermographie infrarouge dans le cadre de relevés aéroportés. Cette comparaison démontre que les résultats

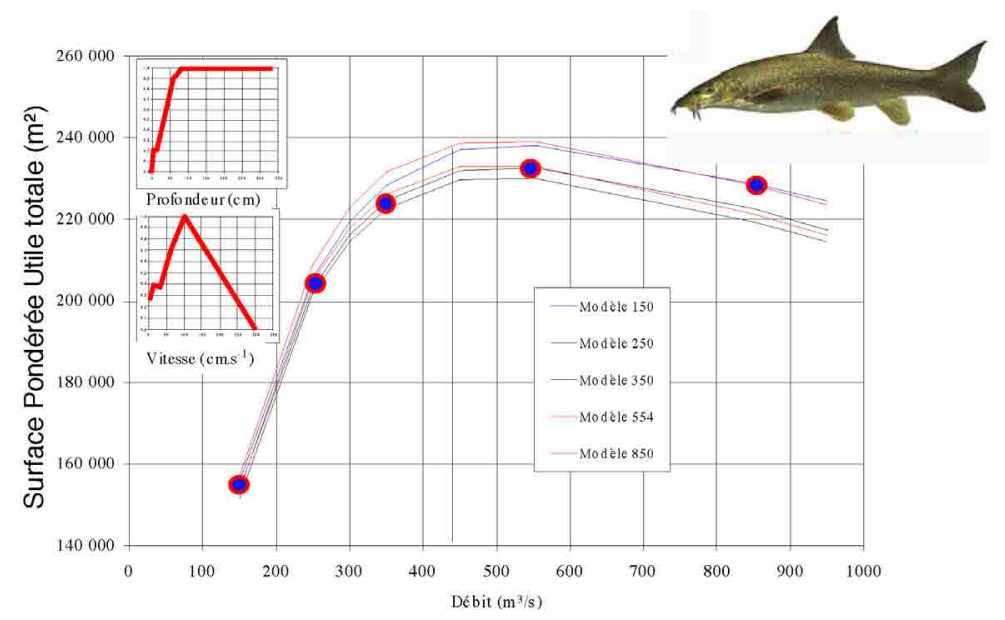

Figure 4 : Évolution de la Surface Pondérée Utile (indice d'habitat favorable potentiel, en $\mathrm{m}^{2}$ ) du barbeau adulte (Barbus barbus, L. 1758) en fonction du débit pour des modèles hydrodynamiques étalonnés à différents débits (150 à $\left.850 \mathrm{~m}^{3} . \mathrm{s}^{-1}\right)$. Les courbes de préférence utilisées pour la profondeur et la vitesse de courant sont présentées à gauche du graphique.

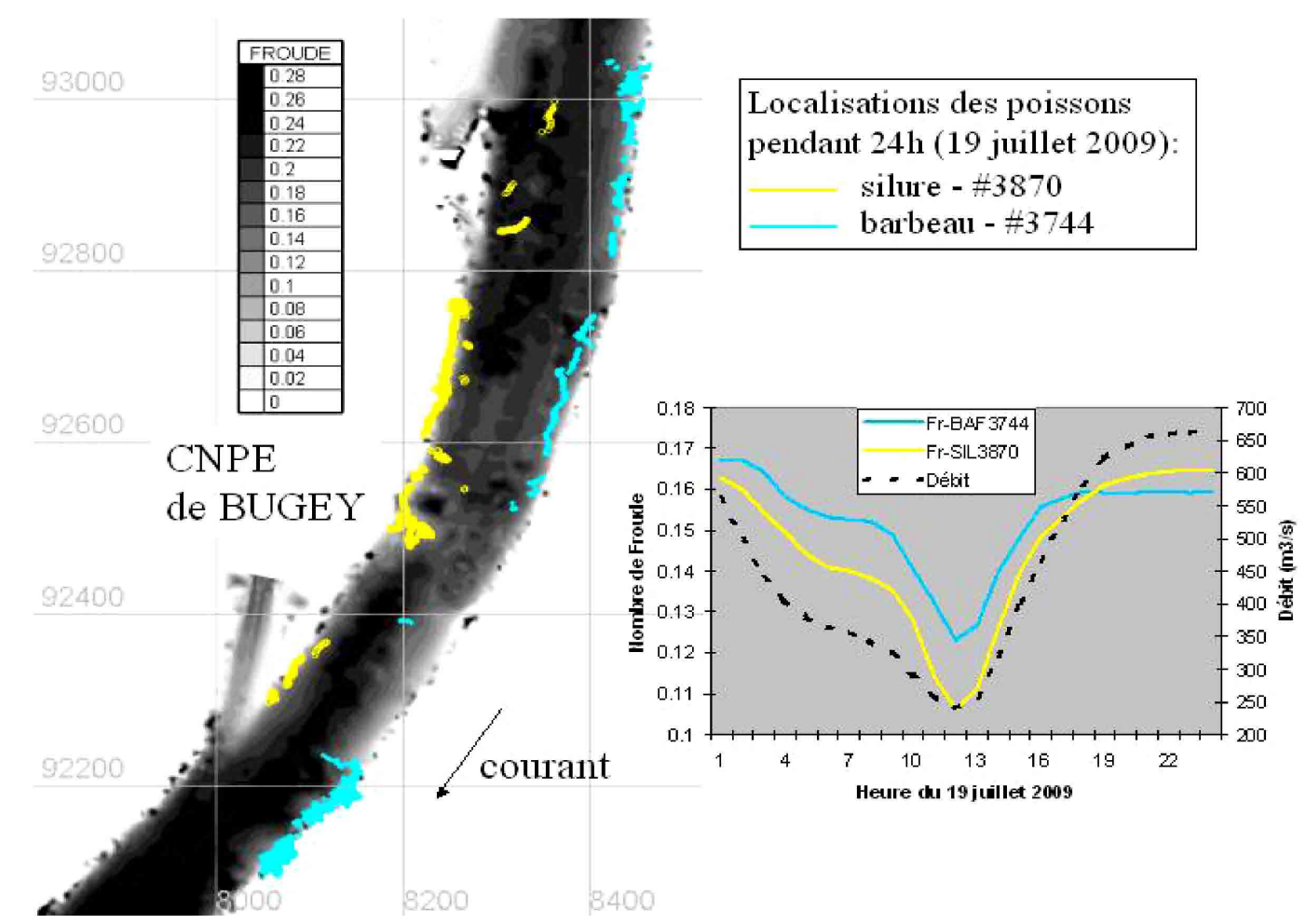

Figure 5 : Exemple de localisation de deux poissons (silure \#3870 (Silurus glanis, L. 1758) et barbeau \# 3744) pendant 24h le 19 juillet 2009 au sein du site d'étude représenté ici par une cartographie du nombre de Froude (dégradé de gris ; adimentionnel) pour le débit moyen de la journée. Le chronogramme des valeurs du nombre de Froude utilisées par les poissons sur $24 \mathrm{~h}$ sont confrontés à la variation concomitante du débit. 
des simulations numériques sont consistants par rapport à la configuration du panache thermique observé in situ.

\section{DISCUSSION}

Les résultats présentés dans cet article ont montré que l'utilisation d'une modélisation en deux dimensions des conditions d'écoulement, d'habitat et de la température de l'eau s'avère un outil performant pour l'analyse des interrelations de divers facteurs environnementaux sur le comportement du poisson dans des milieux complexes tels que le Rhône. Cette approche, peu appliquée à ce jour en France, possède l'avantage marqué de caractériser, de manière continue, l'interaction de paramètres environnementaux sur l'évolution de la structure des communautés aquatiques. En revanche, ces outils de simulation sont déjà largement exploités au Québec, pour le fleuve Saint-Laurent par exemple (Mingelbier et Morin, 2005 [9] ; Morin et al., 2005 [10]). La connaissance des conditions vécues par les poissons dans l'approche comportementale envisagée dans le cadre du projet en cours (projet Bugey 2D) en fait son originalité et sa force. Pour la première fois, les fluctuations des conditions environnementales susceptibles d'influencer les poissons pourront être mises en relation avec leur localisation spatiale. Mais ces outils peuvent également simuler les conditions d'habitat et de température sur des périodes significativement plus longues, notamment celle correspondant au suivi des effets des rejets d'eau chaude sur la structure de la communauté piscicole entamé en 1979. En cela le projet Bugey 2D, via la mise en œuvre d'une modélisation hydrodynamique en $2 \mathrm{D}$, répond à l'une des questions majeures formulées par les experts dans la synthèse du projet Rhône Thermie - phase III (Carrel et al., 2006 [1]).

Les courbes de préférences définies par Lamouroux et al. (1999) [8], lesquelles ont été élaborées à partir de campagnes d'échantillonnage effectuées entre autres dans le Rhône, ont été jugées par ces auteurs comme transférables pour les cours d'eau du sud de la France. Il était donc raisonnable d'utiliser ces courbes dans un premier temps afin d'estimer les surfaces d'habitat favorable pour différentes espèces présentes dans le secteur à l'étude. Toutefois, l'analyse des habitats utilisés par les poissons suivis par télémétrie acoustique, dans le cadre du projet en cours Bugey 2D, permettra certainement une analyse assez fine de la pertinence des simulations d'habitat réalisées ici. Par ailleurs, les premières analyses des enregistrements des comportements des poissons, confirment que le comportement des poissons ne sera pas étudié en tenant compte de leur position dans la colonne d'eau (dimension verticale). Ce constat justifie encore, $a$ posteriori, le choix d'une schématisation bidimensionnelle des écoulements.

Un des axes forts actuels du projet Bugey 2D est la description des trajectoires des poissons dans le temps et dans l'espace. L'une des questions fondamentales associées à cette description des trajectoires des poissons, incluant les moments d'immobilisation, est la recherche d'évènements susceptibles d'expliquer les changements de comportement. L'intérêt de disposer d'une caractérisation des conditions d'habitat et de température de l'eau à des échelles temporelles et spatiales adéquates, et ce, pour une large gamme de débits susceptibles d'être observés dans le secteur d'étude, laisse supposer qu'il sera possible de connaître l'influence de ces deux facteurs environnementaux clefs dans le comportement des poissons.

\section{REMERCIEMENTS}

Nous tenons à remercier EDF (CNPE et DTG-Grenoble), l'Agence de l'Eau RM\&C et l'Union Européenne /FEDER) pour leur collaboration et pour leur soutien financier pour le projet Bugey 2D. Nous remercions également la Compagnie Nationale du Rhône pour nous avoir communiqué les enregistrements de débit et de niveau d'eau au pas de temps horaire à Lagnieu et au droit du CNPE de Bugey. Ces données sont dites «brutes, visuellement contrôlées mais non critiquées et non validées ». Enfin, il importe de souligner ici qu'un tel travail ne peut aboutir que grâce à la collaboration de beaucoup de personnes dévouées, en particulier, Pascal Roger, Raphaël Mons, Julien Bergé et Nicolas Lamouroux.

\section{REFERENCES ET CITATIONS}

[1] Carrel G., Desaint B., Fruget J. F., Khalanski M., Olivier J. M., Poirel A. Et Souchon Y. (2006) - Etude thermique globale du Rhône - Phase III Synthèse et conclusions. $59 \mathrm{p}$

[2] Berge J., Capra H., Ovidio M., Pella H., \& Lamouroux N. (Soumis) - Living with fish in a large river with heterogeneous flow and temperature : an acoustic telemetry experiment.

[3] Reiser D. W., Wesche T. A. \& Estes C. (1989) - Status of instream flow legislation and practices in North America. Fisheries. 14 22-29

[4] Tharme R. E. (2003) - A global perspective on environmental flow assessment: emerging trends in the development and application of environmental flow methodologies for rivers. River Research and Applications. 19 397-441

[5] Hayes J., Hughes N. \& Kelly L. (2007) - Process-based modelling of invertebrate drift transport, net energy intake and reach carrying capacity for drift-feeding salmonids. Ecological Modelling. 207 171-188

[6] Pella H., Capra H., \& Foulard S. (2008) - Développement d'un MNT du haut Rhône à partir de relevés bathymétriques réalisés avec un sondeur multi-faisceaux. Revue Française de Photogrammétrie et de Télédétection. 186 81-86

[7] Capra H., Pella H. \& Oriol E. (2008) - Relevés de la température de l'eau du Rhône au cours de l'été 2008 (profils et enregistrements ponctuels). Rapport Cemagref - EDF. 42 p

[8] Lamouroux N., Capra H., Pouilly M. Et Souchon Y. (1999) - Fish habitat preferences in large streams of southern France. Freshwater Biology. 42 673-687

[9] Mingelbier M. \& Morin J. (2005) - Modélisation numérique $2 D$ de l'habitat des poissons du Saint-Laurent fluvial pour évaluer l'impact des changements climatiques et de la régularisation. Le Naturaliste Canadien. 129 96-102

[10] Morin J., Champoux O., Martin S. \& Turgeon K. (2005) - Modélisation intégrée de la réponse de l'écosystème dans le fleuve Saint-Laurent: Rapport final des activités entreprises dans le cadre du Plan d'étude sur la régularisation du lac Ontario et du fleuve Saint-Laurent. Rapport scientifique SMC Québec - Section Hydrologie RS-108, Environnement Canada, Sainte-Foy, préparé pour le Groupe de travail technique sur l'environnement du Groupe d'étude International sur le lac Ontario et le fleuve Saint-Laurent (Commission Mixte Internationale). $130 \mathrm{p}$ 